\title{
Design Method of Polymer Parameters of ASP Flooding in Second-type Layers
}

\author{
GuangJie Zhao \\ No.1 Oil Production Plant of Daqing Oilfield Company, Daqing, 163000, China \\ email: 43573422@qq.com
}

\begin{abstract}
Keywords: ASP Flooding, Injection Parameters, Specific Design, Control Degree of Chemical Flooding
\end{abstract}

\begin{abstract}
ASP flooding has been extensively used in Daqing Oilfield, but due to heterogeneity of second-type layers and the sameness of injection parameters, different permeability zones have different production status. The study focused on the design and optimization of injection parameters of ASP flooding, for the purpose to improve the matching rate of injection parameters with the target zones and enhance recovery of ASP flooding. The design fully considered the features of oil-bearing zones, permeability distribution, control degree of chemical flooding, well clusters and the relationship between ASP recipe and permeability, and provided the design method of injection parameters for single well. Oilfield application and practice indicated that the method can improve the matching rate of injection parameters of single well with the target zones by $9.8 \%$ and it can be used to realize high efficiency ASP flooding in the future.
\end{abstract}

\section{Introduction}

ASP flooding began in Daqing Oilfield in 1994, and after pilot tests and industrial tests, was put into large-scale application in 2007 and achieved outstanding results [1][2]. But with the extensive use of ASP flooding in second-type layers, emerged the problems such as lateral and vertical heterogeneity of second-type layers and different features of oil-bearing zones among well clusters [3], as well as the sameness of injection parameters, which caused different production status of different permeability zones and low matching rate of injection parameters with target zones and influenced ASP flooding efficiency. Therefore the study is carried out to find the specific design of injection parameters of ASP flooding and provide support for future development and adjustment of the oilfield.

Decades of practice of ASP flooding suggested that ASP injection includes 4 phases [4][5], the pre-slug of polymer as a sacrificial agent to reduce alkali loss and adsorption of surfactant and improve sweep efficiency and plug the high-permeability zones to adjust the injection profiling, the primary-slug and secondary-slug of ASP to reduce interfacial tension and further improve sweep efficiency to displace the moderate-low permeability zones, and post-slug of polymer to protect the ASP system. Each phase plays different roles and the polymer molecular weight and concentration of each phase are designed accordingly different.

\section{Design of Polymer Molecular Weight}

Molecular weight of polymer is designed according to lab analysis on how the polymer system [6] and ASP system matched with the oil-bearing layers [7], as well as considering the permeability distribution and chemical flooding control degree of the block and classification of well clusters and learning from experiences of blocks where polymer flooding has already been applied.

Design of Polymer Molecular Weight based on Permeability Distribution. Core experiment in the lab suggested that polymer molecular weight is closely related with core permeability (Fig.1). In ASP system, the cutoff of permeability allowable for the moderate molecular weight (12 16 million), the high weight (16 19 million) and ultra-high weight (25 million) of polymer to pass through is respectively $110 \mathrm{mD}, 170 \mathrm{mD}$ and $230 \mathrm{mD}$, while in polymer system, the allowable cutoff of permeability is $130 \mathrm{mD}, 180 \mathrm{mD}$ and $280 \mathrm{mD}$ accordingly. Design of polymer for a block should 
guarantee that it is applicable to more than $90 \%$ of the target zones.

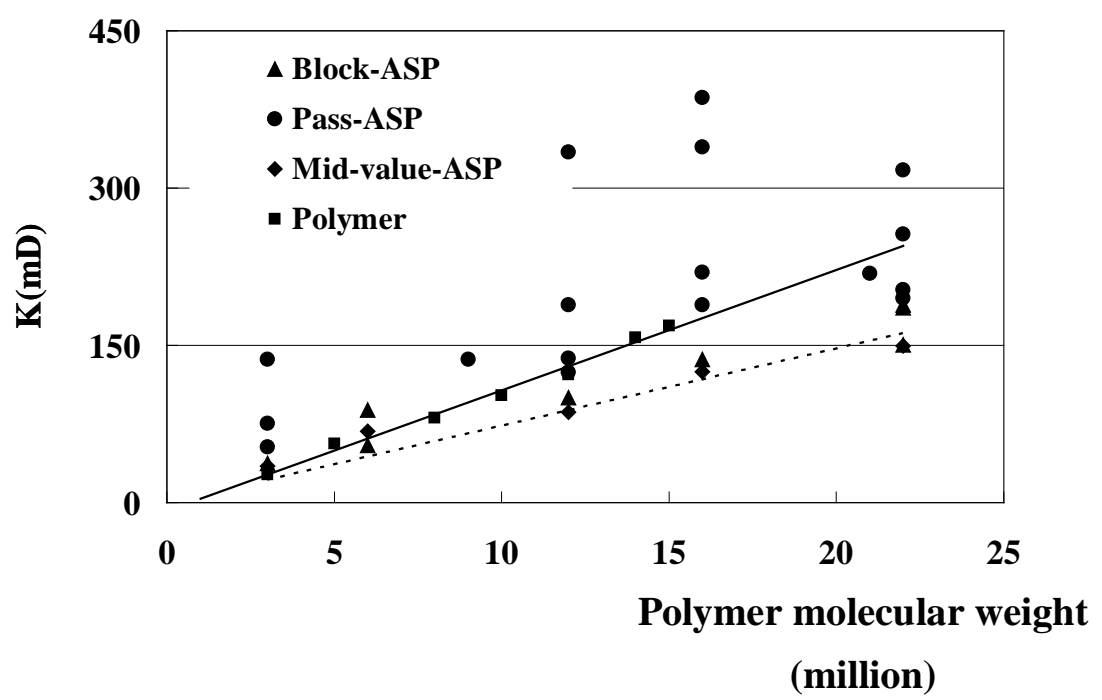

Fig.1 Relationship between polymer molecular weight and core permeability

Design of Polymer Molecular Weight based on Chemical Flooding Control Degree. Oilfield practices and reservoir simulation suggested that [8] chemical flooding has a certain impact on oil displacement efficiency and when chemical flooding control degree is above $70 \%$, recovery is improved better. The purpose of pre-slug of polymer is to adjust and plug the high-permeability zones and the polymer chosen to be used should have as high molecular weight as appropriate.

Design of Polymer Molecular Weight by Analogy Method. Design of polymer molecular weight of a block can count on statistics of permeability distribution, injection status and development effect of other blocks which have similar features of oil-bearing zones as the block, and refer to the median of pore radius that polymer in different systems can pass through (Table 1).

Table 1 Comparison of pore radius that polymer in different systems can pass through

\begin{tabular}{c|c|c|c|c|c|c}
\hline Molecular weight (million) & 10 & 12 & 15 & 16 & 19 & 25 \\
\hline System & 1.04 & 1.11 & 1.24 & 1.31 & 1.43 & 1.69 \\
\hline Polymer solution & 0.94 & 1.03 & 1.17 & 1.21 & 1.33 & 1.55 \\
\hline ASP
\end{tabular}

Refer to Block of Polymer Flooding. Block D has similar second-type layer features with block A, it used moderate molecular weight of polymer and achieved good injectivity and development effect and improved recovery by more than $13 \%$. The moderate molecular weight polymer in the polymer solution can pass through $1.11-1.31 \mu$ median of pore radius, and high molecular weight polymer in ASP system can pass through 1.03-1.21 $\mu$ m median of pore radius, so the design of block A can use high molecular weight polymer.

Refer to Block of ASP Flooding. Block B has similar second-type layer features with block A, it used ultra-high molecular weight polymer in ASP flooding and achieved good injectivity and development effect and improved recovery by more than $20 \%$. Block $\mathrm{C}$ injected moderate and high molecular weight polymer in ASP flooding. According to permeability distribution of block A (Figure 2), the proportion of layers with permeability less than $400 \mathrm{mD}$ in block $\mathrm{A}$ is similar to that of block $\mathrm{C}$ and higher than that of block $\mathrm{B}$, while proportion of layers with permeability more than $700 \mathrm{mD}$ is somewhere in between of block B and block C. The design of ASP slug of block A can use moderate and high molecular weight polymer to avoid blocking the layers and the pre-slug of polymer can use high and ultra-high molecular weight polymer.

Design of Polymer Molecular Weight based on Classification of well Clusters. Second-type layers are heterogeneous and the situation between wells, layers and inside layers differs a lot. Indices such as net pay, channel sand thickness, channel sand connectivity, chemical flooding control degree, and permeability distribution etc. are selected to build a quantified classification standard for wells, and the injection well clusters in the block can be classified into 4 types. According to the chart of matching relationship between ASP system and permeability [7] and 
statistics of actual injection status, Type-I and II wells can use polymer of higher molecular weight and Type-III and IV wells can use lower molecular weight polymer.

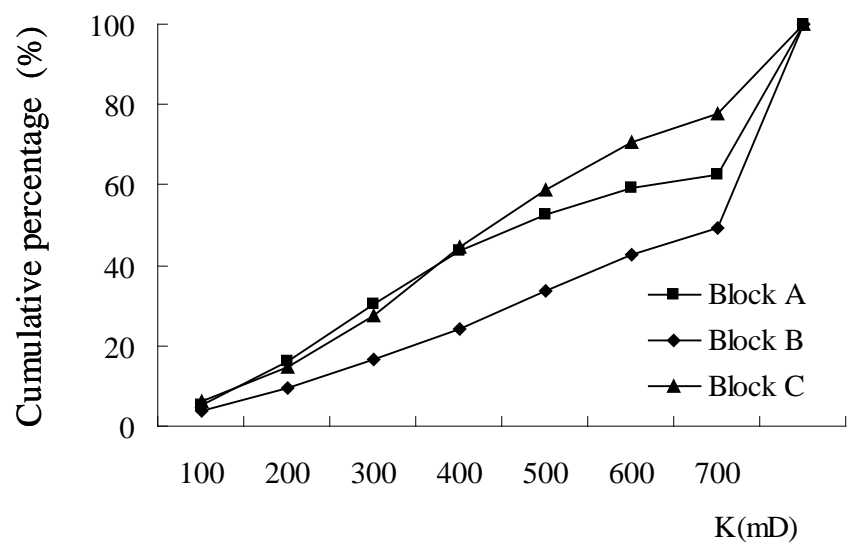

Fig.2 Comparison of permeability distribution

Design of Polymer Molecular Weight based on Static and Dynamic Monitoring of Permissible Injection Pressure. Based on experiences from other injected blocks, polymers of moderate molecular weight and high molecular weight are injected according to permissible injection pressure of wells and by adjusting the process of injection, the injection pressure reached equilibrium, therefore, the molecular weight of polymer for wells can be optimally designed according to permissible injection pressure of well.

\section{Design of Concentration of Polymer}

Injection viscosity is designed according to crude oil viscosity and permeability distribution, and then concentration of polymer solution can be determined by the curve of relationship between viscosity and concentration of ASP system.

Design of Polymer Concentration based on Crude Oil Viscosity. Results of sand tube experiment of ASP compounds of different viscosities suggested that under the same permeability, there is an optimum viscosity other than the assumption that the higher viscosity the better. Experiment of compounds of different permeability suggested that the optimum viscosity for second-type layer $A$ is $20 \sim 30 \mathrm{mPa} \cdot \mathrm{s}$ and for second-type layer $B$ is $10 \sim 20 \mathrm{mPa} \cdot \mathrm{s}$. Plenty of experimental researches[9][10] and reservoir simulation [11] indicated that when the ratio of injection viscosity and crude oil viscosity of heterogeneous layers is 3 , the recovery is not improving as good as before when the ratio is controlled within $2 \sim 4$ which is more reasonable a range. During ASP injection, reservoir resistance coefficient increases and injectivity weakens, and the viscosity of post-slug of polymer is lower than the primary and secondary slugs of ASP. Judging from permeability distribution (Fig. 1) and experiences of blocks which injection have been carried out, the viscosity of pre-slug and primary and secondary slugs for second-type layer A is designed to be 3 times of crude oil viscosity if working underground and viscosity of post-slug is around 2 times of crude oil viscosity. Cored wells proved that viscosity loss of ASP system is about 50\% from well head to the reservoir. Based on the designed viscosity of slugs of each phase, the concentration of polymer solution for each phase can be determined via the curve showing relationship between viscosity and concentration of polymer and ASP systems.

Design of Polymer Concentration based on Permeability. To make the injection system best matching with the target reservoir, the injected polymer concentration can be optimized based on the relationship between ASP system and permeability and according to the main range of permeability of wells.

The main range of permeability refers to the permeability of a well which are ranged orderly according to its values and its corresponded cumulative thickness should reach a certain number to be the main range of distribution of permeability. For instance, when making statistics of permeability of separate layer of a well, $K_{50}$ is the permeability corresponding to the net pay which cumulatively counted $50 \%$ thickness of the well and $\mathrm{K}_{50}$ is $490 \mathrm{mD}$, it means permeability of more 
than $50 \%$ thickness of the well is higher than $490 \mathrm{mD}$. By this method, when the counted cumulative net pay of the well is $60 \%, 70 \%, 80 \%$ and $K_{60}, K_{70}, K_{80}$.

According to statistics of profiling status during injection, the using rate of polymer pre-slug is $74.3 \%$ and using rate of ASP primary and secondary slug is $80.3 \%$ and using rate of polymer post-slug is $76.5 \%$, therefore the main range of permeability of polymer pre-slug is determined as $\mathrm{K}_{70}$ and for ASP primary and secondary slug and polymer post-slug $\mathrm{K}_{80}$.

Based on the main range of permeability, the matching chart of polymer system and ASP system with permeability, the relationship between viscosity and concentration of polymer system and ASP system, and the design result of polymer molecular weight of well, and considering the permissible injection pressure of well, optimally designed the polymer concentration of each phase of slug.

\section{Application Result}

The design method has been put into practice in block $\mathrm{A}$, where the underground crude oil viscosity is $8.3 \mathrm{mPa} \cdot \mathrm{s}$ and well head injection viscosity is designed to be $50 \sim 35 \mathrm{MPa} \cdot \mathrm{s}$, the polymer pre-slug uses ultra-high and high molecular weight polymer and concentration is $1450 \mathrm{mg} / \mathrm{L}$, while the ASP primary and secondary slug uses high and moderate molecular weight polymer and concentration is respectively $2200 \mathrm{mg} / \mathrm{L}$ and $2100 \mathrm{mg} / \mathrm{L}$, the polymer post-slug uses moderate and high molecular weight polymer and concentration is $1400 \mathrm{mg} / \mathrm{L}$. after ASP flooding in the block, the injection pressure built-up steadily and the differences among wells became less obvious, compared with initial injection pressure of ASP primary slug, the pressure increased by 2.3MPa which averagely raised $0.15 \mathrm{MPa}$ per month. The matching rate of injection parameters reached $85.3 \%$ which is improved by $9.8 \%$ compared with the other blocks. The producing degree of reservoir is $79.9 \%$ which is $10.4 \%$ higher than by water flooding. The block achieved outstanding development effect and the cyclic recovery at the time being is $1.83 \%$.

\section{Conclusion}

Design of injection parameters should follow the rule to consider the whole situation first and then make specific design for single well. The specific design for well should consider both sand development and connectivity and the matching between injection system with permeability, and improve the matching of well with the reservoir statically and dynamically. Optimization of the design of injection parameters should combine with actual application in the oilfield, which shall be timely adjusted based on the dynamic performance and well adjustments, to guarantee injection plan is scientific and rational.

\section{References}

[1] Cheng Jiecheng, Wu Junzheng, Wu Di. ASP oil displacement technology [T]. BeiJing: Petroleum Industry Press, 2013.6: 331-361.

[2] Li Huabin. New progress and field test of ASP[T]. BeiJing: Science Press, 2007: 18-19.

[3] Sui Jun. Daqing Oil Field Excellent essays of Core technical talents [T]. BeiJing: Petroleum Industry Press, 2009.9: 609-612.

[4] Yao Shichun, Li Xinfeng etc. Numerical simulation of injection method for alkali-surfactant-poylmer flooding[J]. Henan Petroleum, 2005, 19(2): 43-46.

[5] Yang Chenglin, Zhou Zhengxiang etc.. Optimization of Injection Parameters for lkali-Surfactant-Polymer Flooding [J]. XINJIANG PETROLEUM GEOLOGY, 2007, 28(5): 604-606.

[6] Huang Bin. Study on Matching Polymer with Reservoir and Injection Way [D]. DaQing: Northeast Petroleum University, 2013: 21-22, 42. 
[7] Yang Xingzhou, Huang Bin. Effect of Different Injection Pattern of ASP System on Displacement Efficiency [J]. Contemporary Chemical Industry , 2015, 44(5): 958-961.

[8] Cheng Jiecheng, Wu Junzheng, Junqing Hu. Key theories and technologies for enhanced oil recovery of alkaline-surfactant-polymer flooding[J]. ACTA PETROLEI SINICA, 2014, 35(2): 310-317.

[9] Zhai Ruibin, Cao Tie etc. .The Effect of Chemical Concentration in ASP System on Oil Dis-placing Efficiency [J]. Petroleum Geology \&Oilfield Development in Daqing, 2002, 21(4): 65-67.

[10] Wang Keliang, Liao Guangzhi etc. . The Effect on Oil Displacement Efficiency of Viscosity of Alkaline-Surfactant-Polymer and Polymer Flooding Solutions [J]. Oilfield Chemistry, 2001, 18(4): 354-357.

[11] Yu Shenghong, Yao Shichun, Jiang Jiang. A study an features of oil displacement of alkali-surfactant-polyner flooding[J]. Henan Petroleum, 2005, 19(2): 33-35. 